\title{
Aplicação de um algoritmo genético ao problema de rodízio de tripulações do sistema de transporte público urbano
}

\author{
Leandro do Carmo Martins ${ }^{1}$ e Gustavo Peixoto Silva²
}

\begin{abstract}
Resumo: Este trabalho aborda a resolução do Problema de Rodízio de Tripulações (PRT) de empresas do sistema de transporte público. O PRT consiste em atribuir uma sequência de jornadas de trabalho aos tripulantes de uma empresa para um dado horizonte de planejamento, com o intuito de minimizar seus custos totais. O custo fixo é dado pelo número de tripulações necessárias para realizar todas as jornadas e os custos variáveis correspondem ao total de horas extras ou ociosas, acumuladas por cada tripulação no período. Na resolução deste problema, são consideradas tanto as restrições operacionais quanto as restrições trabalhistas de uma dada empresa. Neste trabalho, o PRT foi resolvido em duas etapas: a primeira consiste em atribuir os dias de folga, minimizando o número de tripulações. A segunda etapa consiste em alocar as jornadas a serem realizadas, minimizando as horas extras e ociosas no período. As duas etapas foram resolvidas utilizando um Algoritmo Genético ainda não aplicado em casos brasileiros. O algoritmo foi desenvolvido para resolver um caso real e seus resultados foram comparados com a solução exata de um modelo de Programação Linear Inteira, mostrando ser competitivo.

Palavras-chave: problema de rodízio de tripulações, programação de tripulações, algoritmos genéticos.
\end{abstract}

\begin{abstract}
This paper addresses the resolution of the Crew Rostering Problem (CRP). The problem consists of assigning duties to the crew members of a company over a given planning horizon, in order to minimize its total costs. The number of crews required to perform all journeys is considered as the fixed costs, while the accumulated overtime hours and idle hours for each crew are the variables costs. In the resolution of this problem, it must be considered the labor laws and the operational constraints of each company. In this paper, we solved the CRP in two stages. In the first of them, we defined the rest period, minimizing the total number of crews. In the second stage, we allocated the duties to be performed by crews, minimizing idle and overtime hours. Both stages were solved using a Genetic Algorithm, a novel CRP approach to Brazilian cases. The algorithm was designed to solve a real case from a company and its results were compared with the exact solutions obtained by an Integer Programming Model, indicating to be competitive.
\end{abstract}

Keywords: crew rostering problem, crew scheduling, genetic algorithms.

\section{INTRODUÇÃO}

O planejamento do Sistema de Transporte Público Urbano é um processo complexo e dividido em etapas, que envolve tanto as empresas responsáveis pela prestação de serviços de transporte público quanto o poder público municipal. Este planejamento é composto, geralmente, pelas seguintes etapas: $i$ ) a definição das linhas de ônibus e seus traçados, ii) a criação dos quadros de horários das linhas para todos os dias da semana, iii) a programação dos veículos que devem realizar as viagens, $i v$ ) a programação diária das tripulações que conduzirão a frota em operação, e v) o rodízio de tripulações ao longo de um dado período.

Dentre as etapas do planejamento, ficam sob a responsabilidade das empresas a programação dos veículos, a programação diária das tripulações e o rodízio das tripulações. Neste contexto, as empresas de transporte público devem executar regularmente um conjunto de viagens contidas no quadro de horários das linhas. Devido à variação da demanda diária por viagens, é definido um quadro de viagens para os dias úteis, um para os sábados e um para os domingos (Mayrink e Silva, 2013).

A realização das viagens previstas para um período de planejamento, normalmente de um mês, leva à definição

\footnotetext{
1 Leandro do Carmo Martins, Departamento de Computação, UFOP. (leandro.cm@live.com)

2 Gustavo Peixoto Silva, Departamento de Computação, UFOP. (gustavo@iceb.ufop.br)
}

Manuscrito recebido em 11/02/2016 e aprovado para publicação em 22/07/2016

Este artigo é parte de TRANSPORTES v. 25, n. 1, 2017. ISSN: 2237-1346 (online). DOI:10.14295/transportes.v25i1.1074 de uma escala de trabalho dos motoristas e cobradores, ou Rodízio de Tripulações. Uma escala ou rodízio é composto por sequências de jornadas diárias que devem ser atribuídas às duplas ou tripulações, compostas por um motorista e um cobrador. Uma jornada diária trata-se de um conjunto de viagens que devem ser realizadas por uma mesma tripulação ao longo de um dia. As jornadas têm duração normal de seis horas e quarenta minutos e pertencem a um turno, que depende de seu horário de início. A duração de uma jornada pode ser inferior ou superior à sua duração normal, resultando em uma jornada com horas extras ou horas ociosas. Além disso, uma jornada pode ser do tipo simples ou dupla pegada. Ao contrário da jornada simples, uma jornada do tipo dupla pegada é aquela na qual ocorre um intervalo superior a duas horas de ociosidade entre duas viagens.

No caso estudado, o dia de trabalho é dividido em quatro turnos de seis horas cada um. O primeiro turno inicia às 4:00 horas, o segundo às 10:00 horas e assim sucessivamente. Cada jornada de trabalho pertence a um único turno, que é aquele no qual se encontra o horário de início da jornada.

A definição da escala mensal de trabalho deve satisfazer as restrições trabalhistas e operacionais da empresa, e ao mesmo tempo, minimizar os custos com o pagamento dos salários fixos e das horas extras. Desta maneira, é possível obter uma carga de trabalho das tripulações mais equilibrada, seja na alocação das folgas, como também com a compensação das horas ociosas, minimizando assim, os custos fixos e variáveis da folha de pagamentos da empresa. Assim, podem ser geradas economias para empresa e um ambiente de trabalho mais saudável com cargas de trabalho mais homogêneas. 
Atualmente, a grande maioria das empresas brasileiras de transporte público utiliza uma escala com pouca variação de jornadas, ou mesmo com a alocação de jornadas fixas ao longo do período. Isso se deve ao baixo índice de utilização de recursos tecnológicos pelas empresas do setor (Leite, 2012). O principal motivo da utilização do sistema de escala fixa deve-se à simplicidade de sua definição, visto que cada tripulação realiza sempre as mesmas jornadas. Além disso, esse esquema permite que os funcionários se familiarizem com as rotas e os seus usuários. Nesse sistema, o critério "tempo de casa" é utilizado para a alocação das tripulações, o que pode gerar certa insatisfação entre os funcionários, pois os novatos na empresa acabam ficando com jornadas menos atrativas do que os funcionários mais antigos.

Outra ineficiência do sistema de jornadas fixas está relacionada ao acúmulo de horas extras ou ociosas que cada tripulação pode ter, uma vez que as mesmas jornadas de trabalho são repetidas pelas tripulações ao longo do horizonte de planejamento. Em contrapartida ao sistema de escala fixa, o Rodízio de Tripulações compreende um método de planejamento das escalas mensais de trabalho que prevê a execução de diferentes jornadas ao longo do período, com o intuito de compensar as horas extras com as horas ociosas de dias posteriores, de uma dada tripulação. Assim, o Problema de Rodízio das Tripulações (PRT) consiste na definição de escalas mensais de trabalho das tripulações, de modo que as leis trabalhistas e operacionais sejam respeitadas, e que sejam gerados rodízios mais equilibrados, reduzindo os custos fixos e variáveis com a mão de obra das empresas.

Ao definir uma escala mensal de trabalho, as seguintes restrições operacionais e trabalhistas devem ser consideradas:

1. O horizonte de planejamento tem início numa segunda-feira e tem a duração de um mês ou sete semanas;

2. Todas as tripulações têm direito a uma folga no domingo a cada sete semanas;

3. As tripulações que fazem dupla pegada em uma semana têm direito de folgar no domingo desta semana;

4. Uma tripulação não pode trabalhar mais do que seis dias consecutivos;

5. No decorrer de todos os dias do horizonte de planejamento, as tripulações cumprem somente um tipo de jornada: i) simples ou ii) duplas pegadas;

6. Dentro de uma mesma semana, as tripulações devem executar jornadas dos dias úteis pertencentes ao mesmo turno de trabalho, exceto para aquelas que realizam dupla pegada;

7. O tempo mínimo de descanso entre o término de uma jornada e o início da próxima jornada deve ser maior ou igual a onze horas;

8. As horas extras de uma tripulação podem ser compensadas com horas ociosas ocorridas no mesmo período de planejamento.

Para minimizar o custo de uma escala é necessário combinar jornadas contendo horas extras com aquelas com ociosidade, o que caracteriza o PRT. Esta prática, denominada Banco de Horas, é prevista na Lei 9.601/98, que permite a compensação de horas extras de uma jornada com horas ociosas de outra jornada realizadas por uma mesma tripulação dentro de um horizonte de planejamento.

Uma solução do PRT pode ser representada por uma matriz na qual as linhas correspondem às diferentes tripulações e as colunas representam os dias do horizonte de planejamento. Cada posição desta matriz armazena a jornada a ser executada pela tripulação no respectivo dia. Caso a tripulação esteja de folga naquele dia, a posição da matriz recebe o valor -1 . Devido a esta representação da solução, é perfeitamente possível empregar um Algoritmo Genético na sua resolução. Sendo assim, este trabalho propõe resolver o Problema de Rodízio de Tripulações por meio da metaheurística Algoritmo Genético. O processo de solução do PRT foi dividido em duas etapas, conforme proposto por Caprara et al. (2003). A primeira etapa consiste definir os dias de folga das tripulações e consequentemente, a quantidade de tripulações necessária para realizar todas as jornadas no período. A segunda etapa consiste em alocar as jornadas que devem ser realizadas por cada tripulação nos seus respectivos dias de trabalho, definido na primeira etapa. Ambas as etapas foram resolvidas utilizando um Algoritmo Genético. A utilização de Algoritmos Genéticos para o Problema de Rodízio de Tripulações é uma abordagem ainda inédita na literatura brasileira, que constitui numa elegante e eficiente técnica de otimização para encontrar soluções de boa qualidade.

O trabalho está dividido da seguinte maneira: na Seção 2 é apresentada uma revisão bibliográfica sobre o Problema de Rodízio de Tripulações. A Seção 3 descreve o Algoritmo Genético implementado para resolver o PRT. Na Seção 4 encontram-se os resultados obtidos nos experimentos computacionais, assim como algumas análises. Finalmente, na Seção 5, são apresentadas as conclusões do trabalho.

\section{REVISÃO BIBLIOGRÁFICA}

No trabalho clássico de Carraresi e Gallo (1984), os autores tratam o PRT por meio da abordagem Multi-level Bottleneck Assignment Problem, e mostram que o problema é NP-completo. No modelo apresentado, é atribuído um peso para cada jornada, representando seu custo. Este peso pode ser simplesmente igual ao tempo de duração da jornada ou pode levar em consideração outros fatores, tais como o atraso do turno, o tempo total decorrido antes do início da jornada, entre outros. O problema de encontrar um equilíbrio das jornadas ao longo de um determinado período é então formulado por meio do seguinte problema de gargalo: minimizar a soma dos pesos das jornadas designadas à tripulação que tem a pior configuração. Os autores propõem um algoritmo para encontrar uma solução sub-ótima que é utilizado para resolver o problema de designação com gargalo referente ao PRT. A metodologia utilizada pelos autores formula o PRT para um horizonte de planejamento de $m$ dias, tendo cada dia uma quantidade $n$ de jornadas. Para encontrar as $m$ sequências de jornadas, o problema é tratado como um problema de fluxo em redes, onde um grafo orientado e valorado de $n$-níveis, $G=(N, A)$, é utilizado. 
Bianco et al. (1992) formulam o PRT por meio de um modelo de Programação Linear Inteira (PLI). Nesse trabalho é descrita uma heurística que utiliza um limitante inferior, proveniente de uma formulação matemática, para reduzir as dimensões do problema. A heurística é denominada HRP, e a cada iteração, o algoritmo resolve um problema de designação multinível com gargalo para o qual é proposta uma nova heurística que produz soluções que convergem para a solução ótima.

Caprara et al. (2003) apresentam modelos matemáticos e algoritmos de solução para uma série de problemas de alocação de pessoal (staff scheduling) decorrentes de aplicações da vida real. Nesses problemas são dadas as jornadas diárias a serem executadas. A duração dos períodos de trabalho e de descanso dos empregados é especificada a priori no horizonte de planejamento, constituindo os padrões de folga a serem considerados. Entretanto, a sequência em que os períodos de trabalho e descanso ocorrem, assim como a atribuição diária de cada jornada, deve ser determinada pelo modelo. O principal objetivo é minimizar o número de funcionários necessários para executar todas as jornadas do horizonte de planejamento. Nos modelos apresentados, o problema é decomposto em duas etapas. Na primeira etapa, são definidas as sequências dos períodos de trabalho e de descanso para cada empregado. Este problema é formulado como um problema de PLI e é encontrada uma solução ótima por meio da técnica branch-and-bound. A segunda etapa é tratada como um problema de viabilidade resolvido heuristicamente por meio de uma sequência de problemas de transporte. Nesta etapa, é associada uma jornada a cada período de trabalho, de modo a garantir a viabilidade e equilibrando a quantidade total de trabalho entre os funcionários. A estratégia adotada no presente trabalho de dividir o PRT em duas etapas foi inspirada na abordagem empregada por Caprara et al. (2003).

Ernst et al. (2004) apresentam uma revisão bibliográfica sobre problemas de programação e rodízio de funcionários (staff scheduling and rostering) de diferentes áreas de trabalho e diferentes métodos de resolução. É abordada uma variedade de áreas de aplicação, desde sistemas de transporte a sistemas de saúde, serviços civis e de emergências, indústrias, turismo, assim como as técnicas de resolução comuns aos problemas. Tais técnicas se baseiam na Inteligência Artificial, Programação por Restrições, abordagens matemáticas e metaheurísticas. Além disso, o trabalho traz um esquema de classificação para descrever problemas de rodízio de funcionários. Os autores têm foco principal nos algoritmos apresentados na literatura para gerar rodízios e programações de pessoal, cobrindo áreas relacionadas, como planejamento da força de trabalho e estimativa do número de funcionários necessários para executar um determinado conjunto de tarefas.

Toffolo et al. (2005) formulam o PRT empregando duas metaheurísticas: Simulated Annealing e Iterated Local Search (ILS), sendo adotado como procedimento de busca local o Método Randômico de Descida. Nesse trabalho, uma estrutura de vizinhança é definida pela troca das jornadas entre duas tripulações. Desta forma, para um referido dia, tenta-se trocar as jornadas entre essas duas tripulações. Uma função de avaliação é construída levando em consideração restrições essenciais, tais como as folgas das tripulações; e restrições não essenciais, que são situações que, apesar de indesejáveis, podem ocorrer na solução, como o excesso de horas extras ou ociosas. Os autores mostram que, para a resolução do PRT, o método heurístico ILS foi mais eficiente do que o Simulated Annealing, encontrando soluções com menor custo em menor tempo de processamento.

Mayrink e Silva (2013) também abordam o PRT em duas etapas, utilizando dois modelos distintos de otimização. Na primeira etapa é desenvolvido um modelo de designação baseado no trabalho de Carraresi e Gallo (1984). Assim, cada nó representa uma jornada diária, e os arcos ligam jornadas de um dia com jornadas do dia seguinte e que podem ser cumpridas por uma mesma tripulação. A cada arco é atribuído um custo em função do acúmulo ou compensação de horas extras com horas ociosas. O problema de designação é resolvido de forma iterativa por meio de modelo de circulação, empregando o algoritmo Out-of-kilter (Ahuja et al., 1993). O modelo tem como objetivo minimizar os custos atribuídos às sequências de jornadas construídas iterativamente. A cada iteração, as jornadas do próximo dia são acrescentadas ao rodízio até formarem o rodízio semanal. Posteriormente, utilizando o mesmo método, o rodízio do período é construído utilizando os rodízios semanais como entrada. Uma vez definidas as sequências de jornadas, é aplicado um modelo de PLI para corrigir possíveis inviabilidades nas folgas. Este segundo modelo faz a alocação de folguistas minimizando assim o total de tripulações na solução.

Boas e Silva (2014) resolvem o PRT por meio de um modelo de PLI. O modelo proposto tem como objetivos minimizar o número de funcionários, compensar horas extras com horas ociosas e minimizar a quantidade total de horas extras e ociosas. Além disso, os autores dividem o problema em três cenários operacionais diferentes, estabelecidos para verificar o impacto dos turnos nos resultados encontrados. O primeiro cenário considera os quatro turnos originais da empresa e os outros dois cenários dividem o dia em dois turnos. Para os três cenários considerados, o modelo se mostrou eficiente para a resolução do PRT ao reduzir consideravelmente o número de tripulações, a quantidade total de horas extras e horas ociosas e a diferença entre a carga de trabalho das tripulações, quando comparado à escala realizada pela empresa. Entretanto, o tempo de processamento é muito elevado para resolver os problemas estudados.

Silva e Prates (2014) tratam o PRT por meio da metaheurística Variable Neighborhood Search (VNS), tendo como método de busca local o Variable Neighborhood Descent (VND). A metaheurística VNS foi implementada em três versões, considerando diferentes estruturas de vizinhança. Na primeira estrutura de vizinhança são fixadas duas tripulações escolhidas aleatoriamente, realizando-se a troca de suas jornadas em $k$ dias diferentes. Na segunda estrutura é fixado um dia, variando os $k$ pares de tripulações que terão suas jornadas trocadas. A terceira estrutura de vizinhança é uma combinação das duas estruturas anteriores. Neste caso, a primeira estrutura de vizinhança é utilizada para gerar um vizinho da solução corrente e a segunda estrutura é utilizada para realizar a busca local. Essa última estrutura de vizinhança mostrou-se mais eficiente na resolução do problema. 


\section{ALGORITMO GENÉTICO APLICADO AO PROBLEMA DE RODÍZIO DE TRIPULAÇÕES}

A resolução do PRT foi dividida em duas etapas, ambas utilizando um Algoritmo Genético (Holland, 1975; Goldberg, 1989).

A Representação da Solução, o Mecanismo de Seleção e as Operações de Crossover são idênticas nas duas etapas. A construção da População Inicial, a operação de Mutação, assim como a Função Objetivo, foram implementadas separadamente para cada etapa, visto que os respectivos objetivos são distintos. A Função Objetivo associa um valor de aptidão a cada indivíduo, ou solução, indicando sua adequação ao problema. Desta forma, os termos Função Objetivo e Função de Aptidão são doravante considerados sinônimos.

\subsection{Representação de uma Solução do PRT}

Para o PRT, um indivíduo, ou solução, é representada por uma matriz bidimensional. Cada coluna da matriz representa um dia do horizonte de planejamento e cada linha representa uma tripulação, que deve realizar as jornadas durante os dias do período planejado. Assim, a matriz tem dimensão $n \times m$, onde as linhas da matriz representam as $n$ tripulações e as colunas os $m$ dias do período. Desta maneira, cada elemento $a_{i j}$ da matriz contém uma referência à jornada a ser executada pela tripulação $i$ no dia $j$, ou -1 caso a tripulação esteja de folga neste dia. Esta representação possibilita aplicar os mecanismos inerentes aos Algoritmos Genéticos.

\subsection{População Inicial}

Uma população trata-se de um conjunto de soluções distintas para um determinado problema. Ela é necessária para que o Algoritmo Genético possa iniciar sua busca. Como na maioria dos casos, a população inicial foi gerada utilizando fatores aleatórios. Isto possibilita a construção de uma população bastante diversificada, o que impede a convergência prematura do método (Talbi, 2009). Após definido um tamanho $t$ da população inicial, é dado início ao procedimento de construção da próxima geração de indivíduos, que criam as soluções e corrigem as inviabilidades por ventura causadas.

\subsubsection{Primeira Etapa da Resolução}

Inicialmente, cada matriz solução é criada com $l$ linhas e $c$ colunas, que representam respectivamente a quantidade de jornadas a serem executadas em um dia útil e a quantidade de dias considerados no período. Como a demanda de viagens do transporte público é maior nos dias úteis do que nos finais de semana, o tamanho da matriz fica definido pela quantidade de jornadas a serem cumpridas nos dias úteis e pela quantidade de dias no horizonte de planejamento. Assim, a matriz é capaz de comportar a alocação de todas as jornadas que devem ser realizadas no período a ser planejado.

Definida a matriz, são distribuídas aleatoriamente as folgas durante todo o período. Para tanto, foram considerados dois padrões de folga: o padrão $5 / 1$ e o padrão $6 / 1$. O padrão de folga $5 / 1$ é caracterizado por cinco dias consecutivos de trabalho com uma folga no sexto dia, e o padrão de folga 6/1 é caracterizado com seis dias consecutivos de trabalho com uma folga no sétimo dia. Como as tripulações do tipo dupla pegada devem folgar aos domingos, foi atribuído o padrão de folga 6/1 às mesmas. Isto garante que todas as tripulações que realizam as jornadas do tipo dupla pegada folguem aos domingos. Já o padrão de folga 5/1 é distribuído ao restante das tripulações, com seus dias iniciais de distribuição escolhidos aleatoriamente. Ambos os padrões de folga respeitam a regra do número máximo de dias trabalhados consecutivamente, mas podem falhar na alocação das folgas aos domingos.

O próximo passo da criação da população inicial é a alocação das folgas naturais que surgem nos sábados e nos domingos. Estas folgas são necessárias para cobrir a quantidade de células da matriz que não são preenchidas por uma jornada nos finais de semana, visto que o número de jornadas a serem executadas é menor do que nos dias úteis. $\mathrm{O}$ total destas folgas é obtido por meio da quantidade de jornadas dos dias úteis, $d u$, menos a quantidade de jornadas a serem cumpridas nos sábados e nos domingos, $d s$ e $d d$, respectivamente. Assim, nos finais de semana temos $d u-d s$ folgas naturais nos sábados e $d u-d d$ folgas naturais nos domingos, que devem ser alocadas às tripulações. Esta alocação é feita aleatoriamente, garantindo sempre as folgas aos domingos para as tripulações que realizam dupla pegada ao longo da semana.

Outra restrição que deve ser respeitada é que cada tripulação deve folgar, pelo menos em um domingo durante um período de sete semanas. Como a alocação das folgas pode gerar rodízios que desrespeitam esta regra trabalhista, um novo procedimento é executado para corrigir as possíveis falhas. Assim, os rodízios que infringem tal regra são deslocados para a direita de forma que ao menos uma folga já alocada coincida com um domingo do período.

Devido às folgas atribuídas ao longo do período, fazse necessário acrescentar uma quantidade $q t f$ de tripulações do tipo folguista. Uma tripulação do tipo folguista deve ser alocada à medida que surge a necessidade de se cobrir as tripulações que encontram-se de folga naquele dia. Essas são tripulações iguais às demais, exceto pelo fato de não pertencerem a um determinado turno. $\mathrm{O}$ total de tripulações deste tipo corresponde ao número máximo de jornadas não atendidas dentre todos os dias do período. O total de folguistas é acrescido ao rodízio, e assim a matriz solução fica com $d u+q t f$ linhas, representando os rodízios das tripulações convencionais e das tripulações folguistas.

No último passo da criação da população inicial da primeira etapa, é aplicado um procedimento para reduzir a quantidade de tripulações do tipo folguista nas soluções. Este procedimento troca aleatoriamente uma jornada alocada para um folguista por uma folga alocada a uma tripulação convencional em um dado dia. Esta troca é realizada somente se todas as restrições forem respeitadas. Este procedimento é executado até que não seja possível realizar mais trocas. Ao final, os rodízios preenchidos apenas com folgas são removidos da matriz solução.

Com a execução de todos os procedimentos descritos acima, a solução é então inserida na população inicial. Esta sequência é executada $t$ vezes, ou seja, até que a população inicial alcance o tamanho desejado. Devido aos fatores de aleatoriedade utilizados na construção das soluções, a população inicial desta etapa é diversificada. 


\subsubsection{Segunda Etapa da Resolução}

Esta etapa da resolução do PRT parte de uma solução gerada anteriormente na qual já estão definidos os dias de folga e a quantidade de tripulações necessárias para realizar todas as jornadas do período planejado.

Esta solução é utilizada como base para a construção da população inicial da segunda etapa. Nesta etapa serão alocadas as jornadas realizadas pelas tripulações durante os dias úteis, sábados e domingos.

Como dados de entrada, tem-se um conjunto de jornadas que devem ser realizadas durante os dias úteis, sábados e domingos pelas tripulações. Para cada jornada é informado o horário de início e a sua duração, além do seu tipo, ou seja, se é uma jornada simples ou uma dupla pegada. A distribuição das jornadas é realizada dia a dia. No primeiro dia do horizonte de planejamento, as jornadas são distribuídas aleatoriamente, sem restrições. A partir das jornadas definidas no primeiro dia, são feitas as alocações nos dias subsequentes. Deste ponto em diante, as restrições 5, 6, 7 e 8 , descritas na Seção 1, devem ser respeitadas.

Para um dado dia (coluna), e para cada tripulação (linha), cria-se um conjunto das jornadas que podem ser alocadas àquela tripulação de acordo com a jornada alocada ao dia anterior. Todas as jornadas que farão parte deste conjunto devem respeitar todas as restrições do problema. Ao final da verificação, para cada tripulação, é obtido um conjunto de jornadas que podem ser alocadas a ela no dia corrente. $\mathrm{O}$ tamanho do conjunto referente a cada tripulação varia de acordo com a quantidade de jornadas possíveis de serem alocadas à mesma sem que qualquer restrição seja violada. Desta forma, inicia-se a alocação a partir das jornadas com mais restrições de alocação, ou seja, aquelas que possuem o menor conjunto de jornadas a serem alocadas no dia em questão. Ao alocar uma jornada, os conjuntos das demais tripulações são atualizados. Ao final da distribuição, tem-se um dia do horizonte de planejamento com suas jornadas e folgas definidas. $\mathrm{O}$ processo se repete até o último dia do período.

Na Figura 1 está representado o processo de alocação de jornadas em um horizonte de planejamento de sete dias, de segunda-feira a domingo, considerando seis jornadas a serem executadas nos dias úteis, cinco jornadas no sábado e quatro jornadas no domingo. Para a execução das seis jornadas são necessárias sete tripulações devido às folgas. Primeiramente, as jornadas são alocadas aleatoriamente no primeiro dia do horizonte de planejamento, representado pela segunda-feira. Posteriormente, cria-se o conjunto de jornadas disponíveis para alocação no dia posterior. No passo $i$ da Figura 1, é selecionado o conjunto mais restritivo do momento (linha 3 em negrito) para iniciar a alocação. Em seguida, uma tarefa é aleatoriamente escolhida no conjunto restritivo de jornadas selecionado, e esta é alocada, vide passo ii. Ao ser alocada a jornada $j 3$, seu conjunto é totalmente descartado na alocação (achuriado no passo $i$ ) e a jornada $j 3$ é removida dos demais conjuntos que a contém. Ainda no passo ii, é selecionado o conjunto mais restritivo do momento (linha 7 em negrito) para continuar a alocação e o processo descrito se repete até que todas as jornadas sejam alocadas. E assim sucessivamente. No passo $v$, ao alocar a jornada $j 2$, a jornada $j 5$ também é imediatamente alocada, uma vez que se torna a única jornada a ser alocada após a alocação de $j 2$, que também faz parte do mesmo conjunto de possíveis alocações.

Realizando-se o processo descrito acima para todos os dias do horizonte de planejamento, obtém-se uma solução viável para o PRT, com os dias de folgas e as jornadas definidas para as tripulações durante o período planejado. Esta sequência é executada $t$ vezes, uma para cada solução, até que a população inicial alcance o tamanho desejado.

\begin{tabular}{|c|c|c|c|c|c|c|c|}
\cline { 2 - 7 } \multicolumn{1}{c|}{} & $\mathbf{S}$ & $\mathbf{T}$ & $\mathbf{Q}$ & $\mathbf{Q}$ & $\mathbf{S}$ & $\mathbf{S}$ & $\mathbf{D}$ \\
\hline $\mathbf{1}$ & $\mathrm{j} 1$ & & -1 & & & & -1 \\
\hline $\mathbf{2}$ & $\mathrm{j} 6$ & & & & & -1 & \\
\hline $\mathbf{3}$ & $\mathrm{j} 4$ & & & & & -1 & \\
\hline $\mathbf{4}$ & $\mathrm{j} 3$ & & & -1 & & & \\
\hline $\mathbf{5}$ & -1 & & & & & & -1 \\
\hline $\mathbf{6}$ & $\mathrm{j} 2$ & -1 & & & & & -1 \\
\hline $\mathbf{7}$ & $\mathrm{j} 5$ & & & & -1 & & \\
\hline
\end{tabular}

\begin{tabular}{|c|c|c|c|c|c|c|c|}
\hline $\boldsymbol{i i i}$ & $S$ & $\mathbf{T}$ & $\mathbf{Q}$ & $\mathbf{Q}$ & $S$ & $S$ & D \\
\hline 1 & j1 & & -1 & & & & -1 \\
\hline 2 & j6 & & & & & -1 & \\
\hline 3 & j4 & j3 & & & & -1 & \\
\hline 4 & j3 & & & -1 & & & \\
\hline 5 & -1 & & & & & & -1 \\
\hline 6 & $\mathrm{j} 2$ & -1 & & & & & -1 \\
\hline 7 & j5 & $\mathrm{j} 4$ & & & -1 & & \\
\hline
\end{tabular}

\begin{tabular}{|l|c|c|c|c|c|c|c|}
\cline { 2 - 7 } \multicolumn{1}{c|}{} & $\mathbf{S}$ & $\mathbf{T}$ & $\mathbf{Q}$ & $\mathbf{Q}$ & $\mathbf{S}$ & $\mathbf{S}$ & $\mathbf{D}$ \\
\hline $\mathbf{1}$ & $\mathrm{j} 1$ & & -1 & & & & -1 \\
\hline $\mathbf{2}$ & $\mathrm{j} 6$ & $\mathrm{j} 2$ & & & & -1 & \\
\hline $\mathbf{3}$ & $\mathrm{j} 4$ & $\mathrm{j} 3$ & & & & -1 & \\
\hline $\mathbf{4}$ & $\mathrm{j} 3$ & $\mathrm{j} 1$ & & -1 & & & \\
\hline $\mathbf{5}$ & -1 & $\mathrm{j} 5$ & & & & & -1 \\
\hline $\mathbf{6}$ & $\mathrm{j} 2$ & -1 & & & & & -1 \\
\hline $\mathbf{7}$ & $\mathrm{j} 5$ & $\mathrm{j} 4$ & & & -1 & & \\
\hline
\end{tabular}

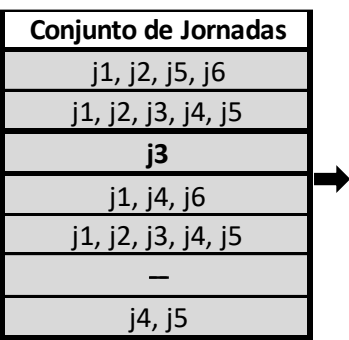

\begin{tabular}{|c|c|c|c|c|c|c|c|}
\cline { 2 - 7 } \multicolumn{1}{c|}{$\boldsymbol{i i}$} & $\mathbf{S}$ & $\mathbf{T}$ & $\mathbf{Q}$ & $\mathbf{Q}$ & $\mathbf{S}$ & $\mathbf{S}$ & $\mathbf{D}$ \\
\hline $\mathbf{1}$ & $\mathrm{j} 1$ & & -1 & & & & -1 \\
\hline $\mathbf{2}$ & $\mathrm{j} 6$ & & & & & -1 & \\
\hline $\mathbf{3}$ & $\mathrm{j} 4$ & $\mathrm{j} 3$ & & & & -1 & \\
\hline $\mathbf{4}$ & $\mathrm{j} 3$ & & & -1 & & & \\
\hline $\mathbf{5}$ & -1 & & & & & & -1 \\
\hline $\mathbf{6}$ & $\mathrm{j} 2$ & -1 & & & & & -1 \\
\hline $\mathbf{7}$ & $\mathrm{j} 5$ & & & & -1 & & \\
\hline
\end{tabular}

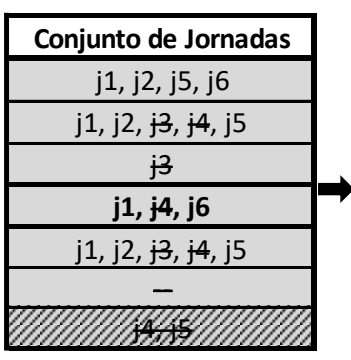

\begin{tabular}{|c|c|c|c|c|c|c|c|}
\cline { 2 - 7 } \multicolumn{1}{c|}{$\boldsymbol{i v}$} & $\mathbf{S}$ & $\mathbf{T}$ & $\mathbf{Q}$ & $\mathbf{Q}$ & $\mathbf{S}$ & $\mathbf{S}$ & $\mathbf{D}$ \\
\hline $\mathbf{1}$ & $\mathrm{j} 1$ & & -1 & & & & -1 \\
\hline $\mathbf{2}$ & $\mathrm{j} 6$ & & & & & -1 & \\
\hline $\mathbf{3}$ & $\mathrm{j} 4$ & $\mathrm{j} 3$ & & & & -1 & \\
\hline $\mathbf{4}$ & $\mathrm{j} 3$ & $\mathrm{j} 1$ & & -1 & & & \\
\hline $\mathbf{5}$ & -1 & & & & & & -1 \\
\hline $\mathbf{6}$ & $\mathrm{j} 2$ & -1 & & & & & -1 \\
\hline $\mathbf{7}$ & $\mathrm{j} 5$ & $\mathrm{j} 4$ & & & -1 & & \\
\hline
\end{tabular}

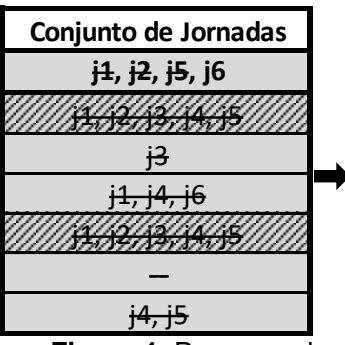

\begin{tabular}{|c|c|c|c|c|c|c|c|}
\hline$v i$ & $S$ & $\mathbf{T}$ & $\mathbf{Q}$ & $\mathbf{Q}$ & $S$ & $S$ & D \\
\hline 1 & j1 & j6 & -1 & & & & -1 \\
\hline 2 & j6 & j2 & & & & -1 & \\
\hline 3 & j4 & j3 & & & & -1 & \\
\hline 4 & j3 & j1 & & -1 & & & \\
\hline 5 & -1 & j5 & & & & & -1 \\
\hline 6 & $\mathrm{j} 2$ & -1 & & & & & -1 \\
\hline 7 & j5 & j4 & & & -1 & & \\
\hline
\end{tabular}

Figura 1. Processo de alocação das jornadas
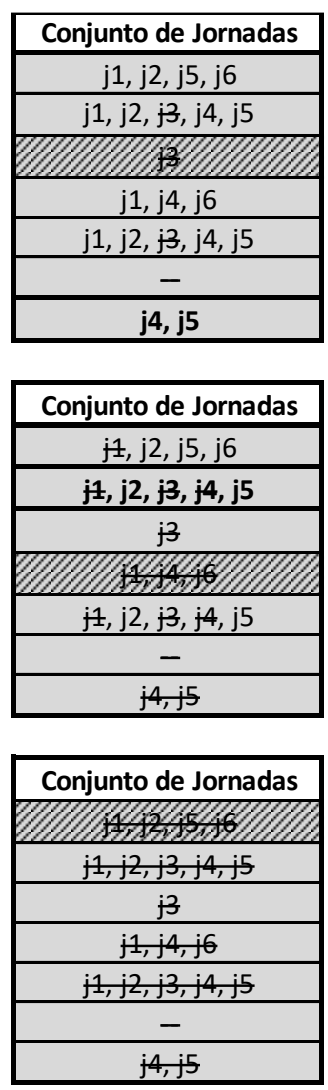
Cada solução a ser inserida na população inicial possui os mesmos dias de folgas definidos na primeira etapa, variando-se apenas as jornadas alocadas em cada dia de trabalho.

Tanto a escolha das jornadas alocadas ao primeiro dia do período quanto a escolha da jornada pertencente ao conjunto de menor cardinalidade são feitas de forma aleatória. Devido aos fatores de aleatoriedade empregados na construção das soluções, a população inicial desta etapa também é diversificada.

\subsection{Mecanismo de Seleção}

A partir da população inicial, o Algoritmo Genético inicia a seleção dos melhores indivíduos, ou seja, daqueles com os menores valores, segundo a função objetivo. Estes indivíduos são utilizados para gerar descendentes (filhos) por meio da operação de crossover. Em seguida os filhos são submetidos à operação de mutação. No crossover, dois pais são necessários para efetuar a recombinação de seus genes e gerar dois filhos distintos. Para definir os pais, foram utilizados os mecanismos de seleção por torneio e o método de seleção Stochastic Universal Sampling (Talbi, 2009). Na seleção por torneio, $k$ indivíduos são escolhidos aleatoriamente. Dentre estes indivíduos, é escolhido aquele que apresenta o melhor valor da função objetivo, a ser descrita a seguir. Para o PRT foram considerados três indivíduos $(k=3)$. O método de seleção Stochastic Universal Sampling funciona de modo que a população esteja disposta em um gráfico de pizza, sendo que para cada indivíduo é atribuído um espaço proporcional à sua aptidão (Whitley, 1994). Uma roleta exterior é colocada sobre o gráfico de pizza com $k$ ponteiros igualmente espaçados. Com uma única rodada da roleta são selecionados, simultaneamente, todos os $k$ indivíduos da população que foram apontados pelos ponteiros. Cada mecanismo de seleção é responsável pela escolha de metade das soluções a serem submetidas ao crossover.

\subsection{Operação de Crossover}

Após a seleção dos pais, seus genes são combinados de forma a gerar dois novos cromossomos filhos, tendo cada um deles um conjunto de genes dos cromossomos pais. Para tanto, foi utilizado o operador crossover OX, adaptado ao problema. Este operador é caracterizado por escolher aleatoriamente dois pontos de corte em cada cromossomo pai e, em seguida, recombinar as parcelas limitadas pelos pontos de cortes, gerando dois novos cromossomos filhos. Os novos cromossomos herdam as faixas de jornadas compreendidas entre os cortes de seus respectivos pais (Talbi, 2009). Os cortes são realizados verticalmente, permitindo assim que os indivíduos possam ser cruzados e que as jornadas pré-definidas para cada dia do horizonte de planejamento sejam mantidas. Cruzamentos de matrizes com números diferentes de rodízios (tripulações ou linhas) podem ser realizados, visto que os novos indivíduos gerados passam a ter a mesma quantidade de linhas da maior matriz, e no mínimo, a mesma quantidade de rodízios da menor matriz solução. O cruzamento é finalizado completando a(s) parte(s) proveniente(s) da menor matriz, na nova solução, com valores que representam folgas e não alteram a execução das jornadas.
O processo de crossover é exemplificado nas Figuras 2 e 3. Neste exemplo, as soluções pais representam um horizonte de planejamento de uma semana, contendo, respectivamente, cinco e sete rodízios. Os valores negativos representam as folgas e os valores positivos representam as jornadas a serem executadas. Os dois pontos de cortes escolhidos aleatoriamente determinam as faixas de jornadas que cada solução descendente herdará de seus pais. Após o cruzamento, as novas soluções passam a ter sete rodízios, visto que é a quantidade de rodízios da maior solução. As jornadas que completam as novas soluções são preenchidas com um valor negativo, representando uma folga, de modo que não altere a execução das jornadas. A seguir, cada nova solução é submetida aos procedimentos que reparam inviabilidades que puderam ser causadas pelo crossover.

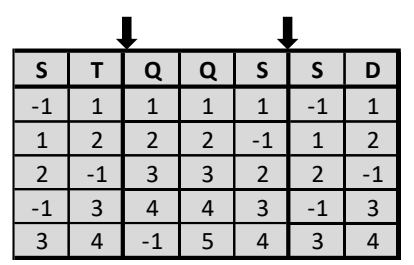

\begin{tabular}{|c|c|c|c|c|c|c|}
\hline $\mathbf{S}$ & $\mathbf{T}$ & $\mathbf{Q}$ & $\mathbf{Q}$ & $\mathbf{S}$ & $\mathbf{S}$ & $\mathbf{D}$ \\
\hline 1 & -1 & 1 & 1 & 1 & 1 & 1 \\
\hline 2 & 1 & 2 & 2 & 2 & 2 & -1 \\
\hline-1 & 2 & 3 & 3 & 3 & -1 & 2 \\
\hline 3 & 3 & -1 & 4 & 4 & 3 & 3 \\
\hline 4 & 4 & 4 & -1 & 5 & 4 & 4 \\
\hline 5 & 5 & 5 & 5 & 6 & -1 & 5 \\
\hline-1 & 6 & 6 & 6 & 7 & 5 & -1 \\
\hline
\end{tabular}

Figura 2. Soluções pais submetidas ao crossover
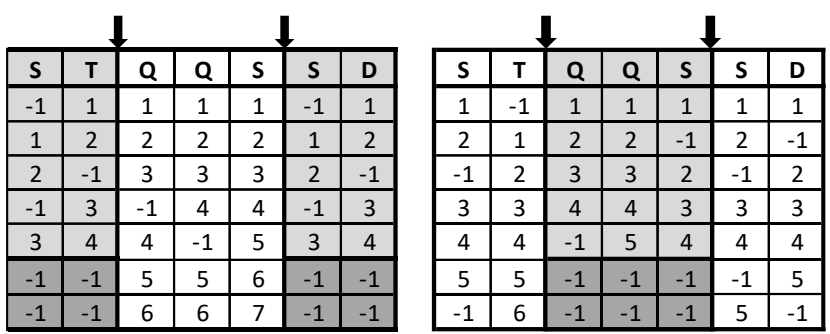

Figura 3. Soluções filhas geradas pelo crossover

\subsection{Operação de Mutação}

A operação de mutação é realizada de acordo com uma dada probabilidade, definida a priori. Esta operação difere para cada uma das etapas da resolução do PRT.

\subsubsection{Primeira Etapa da Resolução}

Na primeira etapa do PRT, o processo de mutação consiste na troca de uma folga por uma jornada de trabalho em um dado dia. Esta operação é exemplificada na Figura 4.

\begin{tabular}{|c|c|c|c|c|c|c|}
\hline $\mathbf{S}$ & $\mathbf{T}$ & $\mathbf{Q}$ & $\mathbf{Q}$ & $\mathbf{S}$ & $\mathbf{S}$ & $\mathbf{D}$ \\
\hline-1 & 1 & 1 & 1 & 1 & -1 & 1 \\
\hline 1 & 2 & 2 & 2 & -1 & 1 & 2 \\
\hline 2 & -1 & 3 & 3 & 2 & 2 & -1 \\
\hline-1 & 3 & 4 & 4 & 3 & -1 & 3 \\
\hline 3 & 4 & -1 & 5 & 4 & 3 & 4 \\
\hline
\end{tabular}$\Rightarrow$\begin{tabular}{c|c|c|c|c|c|c|c|}
\hline $\mathbf{S}$ & $\mathbf{T}$ & $\mathbf{Q}$ & $\mathbf{Q}$ & $\mathbf{S}$ & $\mathbf{S}$ & $\mathbf{D}$ \\
\hline-1 & 1 & 1 & 1 & 1 & -1 & 1 \\
\hline 1 & 2 & 2 & 2 & 3 & 1 & 2 \\
\hline 2 & -1 & 3 & 3 & 2 & 2 & -1 \\
\hline-1 & 3 & 4 & 4 & -1 & -1 & 3 \\
\hline 3 & 4 & -1 & 5 & 4 & 3 & 4 \\
\hline
\end{tabular}

Figura 4. Processo de mutação para a primeira etapa do PRT

\subsubsection{Segunda Etapa da Resolução}

Na segunda etapa do PRT, o processo de mutação é caracterizado pela troca de jornadas em um dia entre duas tripulações que não estão de folga, escolhidas aleatoriamente. Esta operação é apresentada na Figura 5. 


\begin{tabular}{c|c|c|c|c|c|c|}
\hline $\mathbf{S}$ & $\mathbf{T}$ & $\mathbf{Q}$ & $\mathbf{Q}$ & $\mathbf{S}$ & $\mathbf{S}$ & $\mathbf{D}$ \\
\hline-1 & 1 & $\mathbf{1}$ & 1 & 1 & -1 & 1 \\
\hline 1 & 2 & 2 & 2 & -1 & 1 & 2 \\
\hline 2 & -1 & 3 & 3 & 2 & 2 & -1 \\
\hline-1 & 3 & 4 & 4 & 3 & -1 & 3 \\
\hline 3 & 4 & -1 & 5 & 4 & 3 & 4 \\
\hline
\end{tabular}$\Rightarrow$\begin{tabular}{|c|c|c|c|c|c|c|}
\hline S & $\mathbf{T}$ & $\mathbf{Q}$ & $\mathbf{Q}$ & $\mathbf{S}$ & $\mathbf{S}$ & $\mathbf{D}$ \\
\hline-1 & 1 & 4 & 1 & 1 & -1 & 1 \\
\hline 1 & 2 & 2 & 2 & -1 & 1 & 2 \\
\hline 2 & -1 & 3 & 3 & 2 & 2 & -1 \\
\hline-1 & 3 & 1 & 4 & 3 & -1 & 3 \\
\hline 3 & 4 & -1 & 5 & 4 & 3 & 4 \\
\hline
\end{tabular}

Figura 5. Processo de mutação para a segunda etapa do PRT

\subsection{Função Objetivo}

A Função Objetivo (FO) associa a cada solução um valor real que representa a qualidade ou aptidão (fitness) da solução, por isto também é dita Função de Aptidão. A Função Objetivo (ou de Aptidão) também difere para cada uma das etapas do PRT.

\subsubsection{Primeira Etapa da Resolução}

Para o cálculo da FO de uma solução da primeira etapa, foram consideradas seis características de grande importância para o problema:

- qtc e qtf: a quantidade de tripulações convencionais e de tripulações do tipo folguista, respectivamente;

- qfp: número de folgas fora dos padrões especificados, $5 / 1$ e $6 / 1$;

- qftf: número de ocorrências de uma jornada de trabalho entre duas folgas;

- qdnf: quantidade de rodízios sem a ocorrência de folgas no domingo;

- qf7d: quantidade de ocorrência de sete ou mais dias trabalhados consecutivamente.

Os seis itens que caracterizam uma solução são multiplicados pelos seus respectivos coeficientes $w_{1}, w_{2}, w_{3}, w_{4}$, $w_{5}$ e $w_{6}$, utilizados para representar a qualidade de uma solução do ponto de vista prático. Os itens $q d n f$ e $q f 7 d$ fazem parte das leis trabalhistas e devem ser necessariamente satisfeitas pelas soluções (hard constraints). Desta maneira, seus coeficientes receberam valores elevados. Os demais itens possuem coeficientes com valores menores por representarem restrições de qualidade (soft constraints). O custo de uma solução $s$, Custo Fasel $_{(}(s)$, é dado pelo somatório da quantidade de tripulações convencionais e folguistas necessárias (trips de $s$ ), e pelos itens ponderados de cada tripulação, conforme Equação (1).

$$
\operatorname{Custo}_{\text {Fasel }}(s)=\sum_{i \in \text { trips de s }} \begin{gathered}
\left(w_{1} \times q t c_{i}+w_{2} \times q t f_{i}+\right. \\
w_{3} \times q f p_{i}+w_{4} \times q f t f_{i}+ \\
\left.w_{5} \times q d n f_{i}+w_{6} \times q f 7 d_{i}\right)
\end{gathered}
$$

A Equação (1) representa a Função Objetivo utilizada na implementação da primeira etapa da metaheurística. Os valores considerados para os coeficientes $w_{1}, w_{2}, w_{3}, w_{4}, w_{5}$ e $w_{6}$ foram 100, 500, 10, 50, 700 e 700, respectivamente. Estes valores foram obtidos empiricamente em função do impacto causado nas soluções e na violação das restrições trabalhistas e operacionais das empresas.

\subsubsection{Segunda Etapa da Resolução}

O objetivo da segunda etapa do PRT é realizar a alocação das jornadas às tripulações, respeitando as folgas alo- cadas na primeira etapa. Nestas jornadas estão incluídas diversas informações que as caracterizam, tais como seu turno, o número de horas extras ou ociosas, entre outras. Para o cálculo da FO da segunda etapa do PRT, são considerados o número de horas extras e horas ociosas acumuladas pelas tripulações durante o horizonte de planejamento.

Cada rodízio, ou seja, cada sequência de jornadas contida na solução, e que deve ser realizada por uma tripulação, é formada por um conjunto de jornadas e dias de folga. Ao final do período, tem-se um número de horas extras, $n H e$, e de horas ociosas, $n H o$, para cada rodízio. $\mathrm{O}$ Banco de Horas, mencionado na Seção 1, é utilizado para compensar $n H e$ com $n H o$. A compensação de um rodízio $r$ é realizada por meio da Equação (2).

$$
\text { compensacao }(r)=n H e-n H o
$$

Como resultado da compensação, obtém-se um valor positivo de horas extras, um valor negativo com as horas ociosas, ou um valor nulo quando o número de horas extras for igual ao número de horas ociosas. Isto caracteriza cada rodízio de acordo com sua compensação resultante.

Uma vez que cada rodízio da solução é avaliado pela sua função de compensação (2), deve-se então obter um valor que represente toda a solução. Assim, realiza-se um somatório das compensações positivas e um somatório, em módulo, das compensações negativas, obtendo-se dois novos valores, sumHe e sumHo, respectivamente. Os rodízios que resultam em uma compensação de valor nulo são desconsiderados, visto que seu somatório também geraria um valor nulo, que não interfere no valor final da Função Objetivo.

Uma vez calculados os somatórios das compensações positivas e negativas, obtém-se seu valor médio entre seus rodízios, encontrado por meio da divisão dos valores sumHe e sumHo pela quantidade de rodízios de compensações positivas e negativas, respectivamente. Posteriormente, calcula-se seus respectivos Desvios Padrões Amostrais, $d p H e$ e $d p H o$. A inclusão destes novos dados na FO leva a uma distribuição mais homogênea das cargas horárias de trabalho entre os rodízios.

Por fim, com os Desvios Padrões e os somatórios das compensações calculados, obtem-se a FO para a segunda etapa do PRT. Cada dado considerado é multiplicado pelo seu coeficiente $\alpha_{1}, \alpha_{2}, \alpha_{3}$ e $\alpha_{4}$, utilizados para representar a qualidade de uma solução do ponto de vista prático. Os valores considerados para estes coeficientes foram unitários. A Equação (3) representa a Função Objetivo utilizada na implementação da segunda etapa da metaheurística.

$$
\text { Custo }_{\text {Fase }}(s)=\alpha_{1} \times \text { sumHe }+\alpha_{2} \times \text { sumHo }+\alpha_{3} \times d p H e+\alpha_{4} \times d p H o
$$

\subsection{Seleção da Nova População}

Uma vez selecionadas as soluções pais por meio da seleção por torneio e Stochastic Universal Sampling, são geradas as soluções filhas por meio da operação de crossover. São criadas $2 n$ soluções a partir das $n$ soluções da população inicial ou anterior. Essas soluções são submetidas ao processo de mutação e avaliação, e posteriormente são inseridas na nova população que continuará o processamento do Algoritmo Genético. 
O método de seleção por torneio, $\operatorname{com} k=3$, foi novamente utilizados para selecionar dentre os $2 n$ indivíduos, os $n$ indivíduos que farão parte da nova população. Para cada geração, uma nova população é criada a partir dos métodos mencionados anteriormente.

\section{EXPERIMENTOS COMPUTACIONAIS}

Para testar a metaheurística Algoritmos Genéticos desenvolvida para resolver o PRT, foram utilizados dados reais de uma empresa de transporte público que opera na cidade de Belo Horizonte - MG. O algoritmo foi desenvolvido na linguagem $\mathrm{C}++$ e os experimentos foram realizados em um computador com processador Intel(R) Core(TM) i72600, 8 GB de memória RAM e Sistema Operacional Windows 7 .

\subsection{Dados de Entrada}

Foram utilizados os seguintes dados para alimentar o modelo de otimização: a quantidade de jornadas que devem ser executadas nos dias úteis, nos sábados e nos domingos com os seus respectivos horários de início e de término. Assim, para cada jornada de trabalho, foi possível calcular o seu tempo de duração, sua quantidade de horas extras ou ociosas, sua classificação como jornada simples ou dupla pegada e seu turno. Os dados referentes à empresa são apresentados na Tabela 1 e considerou-se um horizonte de planejamento de sete semanas. A Tabela 1 apresenta, para cada tipo de dia, o total de jornadas, o número de jornadas que são do tipo dupla pegada, o total das horas extras (HE) e de horas ociosas (HO) contidas no conjunto de todas as jornadas.

Foi considerada a restrição operacional de que as tripulações devem se manter em um único turno. Desta forma, o problema foi dividido em quatro turnos independentes gerando quatro novos subproblemas independentes: um para cada turno. Esta divisão possibilitou a comparação dos resultados obtidos neste trabalho com aqueles apresentados por Boas e Silva (2014) que empregaram métodos exatos.

Tabela 1. Quantidade de jornadas e suas características

\begin{tabular}{ccccc}
\hline $\begin{array}{c}\text { Dias da } \\
\text { Semana }\end{array}$ & $\begin{array}{c}\text { Total de } \\
\text { Jornadas }\end{array}$ & $\begin{array}{c}\text { Dupla } \\
\text { Pegada }\end{array}$ & HE & HO \\
\hline Dia Útil & 104 & 4 & $62: 46$ & $78: 36$ \\
Sábado & 70 & 0 & $45: 37$ & $26: 54$ \\
Domingo & 53 & 0 & $27: 41$ & $16: 01$ \\
\hline
\end{tabular}

Com a divisão do problema, foram obtidas novas quantidades de jornadas a serem executadas nos dias úteis, sábados e domingos, cada qual relacionada ao seu respectivo turno. Assim, cada subproblema foi resolvido independentemente. Estes dados são apresentados na Tabela 2.

Tabela 2. Quantidade de jornadas para cada turno T1, T2, T3 e

\begin{tabular}{ccccc}
\multicolumn{5}{c}{ T4, respectivamente } \\
\hline Turno & Dia Útil & Sábado & Domingo & $\begin{array}{c}\text { Dupla } \\
\text { Pegada }\end{array}$ \\
\hline T1 & 49 & 28 & 20 & 4 \\
T2 & 38 & 26 & 20 & 0 \\
T3 & 12 & 11 & 10 & 0 \\
T4 & 5 & 5 & 3 & 0 \\
\hline Total & 104 & 70 & 53 & 4 \\
\hline
\end{tabular}

A fim de medir a eficácia do Algoritmo Genético, foram realizados testes computacionais variando-se seus parâmetros para escolher aqueles que gerassem as melhores soluções. Dentre os parâmetros do Algoritmo Genético, foram calibrados o tamanho da população e a quantidade de gerações. A taxa de crossover e de mutação foram definidas a priori, a partir de pesquisas bibliográficas, e seus valores foram de $50 \%$ e $1 \%$, respectivamente. Os valores dos parâmetros foram escolhidos de acordo com o tamanho de cada turno. Assim, o tamanho da população foi de 800, 1200, 1600 e 2000 indivíduos para T1 e T2, e de 100, 150, 200 e 250 para T3 e T4. A quantidade de gerações foi de 1.000 , 1.500 e 2.000 para T1 e T2, e 250, 500 e 750 para T3 e T4. Desta forma, foram consideradas 12 combinações de parâmetros para cada subproblema. Como a condição de parada, para cada conjunto de parâmetros, foi o número de gerações, o tempo de processamento variou em função deste parâmetro, assumindo valores dentro do intervalo de 45 a 120 minutos.

Para cada etapa da resolução do problema, os parâmetros foram calibrados independentemente, uma vez que a função objetivo difere de uma etapa para outra. A partir da solução da primeira etapa foram calibrados os parâmetros da segunda etapa. A calibração feita de forma independente possibilitou obter uma solução final de melhor qualidade para o problema, uma vez que na primeira etapa são definidos os padrões de folga e o número mínimo de tripulações, e na segunda etapa é minimizada a quantidade de horas extras e ociosas da escala.

\subsection{Resolução do Problema}

Utilizando os valores definidos anteriormente para a taxa de crossover e mutação, foram executados testes computacionais para encontrar os melhores valores para o tamanho da população e a quantidade de gerações. Para cada uma das 12 combinações, foram realizadas 5 execuções, e a partir destas, foram selecionados os números de gerações que apareceram nas 4 melhores soluções obtidas. Para cada uma das 4 combinações selecionadas, com o melhor número de gerações fixado, foram executados outros 5 testes variando-se o tamanho das populações. A escolha da melhor combinação foi realizada pelo teste estatístico de Kruskal e Wallis (1952).

No teste de Kruskal e Wallis, os valores da FO das 5 execuções de cada combinação são colocados em ordem crescente e para cada resultado é atribuído um valor de classificação, que varia de 1 até a quantidade de soluções existentes (no caso igual a 20). Em caso de empate entre duas ou mais soluções, o valor de classificação atribuído às mesmas é a média dos valores de classificação que seriam atribuídos na ordem crescente às soluções, caso não houvesse o empate. Depois de ordenados, os valores de classificação são somados para cada combinação, e a partir deles, calcula-se sua média. A combinação com a menor média é escolhida como a melhor combinação de parâmetros, visto que obteve as soluções com as menores classificações em média, ou seja, as soluções com menores FO. O tamanho da população e o número de gerações escolhidos a partir das metodologias mencionadas acima, nas etapas 1 e 2, são apresentados na Tabela 3, para cada turno. 
Tabela 3. Tamanho da população e número de gerações adotados nas etapas 1 e 2 para os turnos (subproblemas) T1, T2, T3 e T4

\begin{tabular}{ccc|cc}
\hline & \multicolumn{2}{c|}{ Etapa 1 } & \multicolumn{2}{c}{ Etapa 2 } \\
\hline Turno & Tamanho da população & Número de gerações & Tamanho da população & Número de gerações \\
\hline T1 & 1.200 & 1.500 & 2.000 & 2.000 \\
T2 & 2.000 & 1.500 & 1.200 & 2.000 \\
T3 & 100 & 500 & 250 & 750 \\
T4 & 200 & 500 & 250 & 750 \\
\hline
\end{tabular}

Tabela 4. Resumo dos resultados obtidos para os subproblemas

\begin{tabular}{cccccccc}
\hline Turno & DU & SAB & DOM & Tripulações & Horas Extras & Horas Ociosas & DP \\
\hline T1 & 49 & 28 & 20 & 50 & $00: 00$ & $1.162: 00$ & 137,2 \\
T2 & 38 & 26 & 20 & 41 & $729: 52$ & $00: 00$ & 30,3 \\
T3 & 12 & 11 & 10 & 14 & $225: 52$ & $00: 00$ & 4,13 \\
T4 & 5 & 5 & 3 & 6 & $00: 00$ & $135: 13$ & 1,9 \\
\hline Total & 104 & 70 & 53 & 111 & $955: 44$ & $1.297: 13$ & - \\
\hline
\end{tabular}

Na Tabela 4 é apresentado o resumo dos melhores resultados obtidos para cada subproblema. A Tabela 4 contém, para cada turno, a quantidade de jornadas para os dias úteis, sábados e domingos, a quantidade de tripulações necessária para executá-las, a quantidade de horas extras e ociosas acumuladas no período de um mês e o Desvio Padrão Amostral - DP (em minutos), refletindo a homogeneidade da distribuição das tarefas às duplas.

A Tabela 4 mostra que em todos os turnos foram encontradas as compensações ótimas, ou seja, foi realizada toda a compensação possível entre horas extras e ociosas. As horas extras foram zeradas nos turnos T1 e T4 e as horas ociosas foram zeradas nos turnos T2 e T3.

Na Tabela 5 é apresentado o melhor resultado obtido pelo Algoritmo Genético juntamente com a solução realizada pela empresa e pelo modelo exato proposto por Boas e Silva (2014). Nesta Tabela, para cada alternativa de solução é apresentado o número de tripulações e o total de horas extras e ociosas.

Tabela 5. Resultados obtidos com o AG, a escala da empresa e por um modelo exato

\begin{tabular}{cccc}
\hline $\begin{array}{c}\text { Alternativa de } \\
\text { Solução }\end{array}$ & Tripulações & $\begin{array}{c}\text { Horas } \\
\text { Extras }\end{array}$ & $\begin{array}{c}\text { Horas } \\
\text { Ociosas }\end{array}$ \\
\hline Algoritmo Genético & $\mathbf{1 1 1}$ & $\mathbf{9 5 5 : 4 4}$ & $\mathbf{1 . 2 9 7 : 1 3}$ \\
Empresa & 244 & $1.106: 39$ & $1.695: 52$ \\
Boas e Silva (2014) & 110 & $955: 44$ & $1.297: 13$ \\
\hline
\end{tabular}

Considerando o número de tripulações, o Algoritmo Genético obteve um resultado bastante satisfatório comparado à escala realizada pela empresa, alcançando uma redução de $55 \%$ do número de condutores. Comparando com os resultados obtidos por Boas e Silva (2014), o Algoritmo Genético também se mostra bastante competitivo, visto que resultou na quantidade ótima de horas extras e horas ociosas, e na diferença de apenas 1 tripulação $(0,91 \%)$ em relação à solução ótima obtida por um modelo de Programação Linear Inteira. A comparação realizada entre os dois métodos visa destacar a qualidade da solução encontrada pelo método proposto, visto que o modelo exato de Boas e Silva (2014) encontra a solução ótima para cada subproblema. Por outro lado, o modelo exato apresentou um tempo de processamento de aproximadamente 8 horas para os turnos T1 e T2, e é limitado na resolução de problemas maiores, o que não acontece com o Algoritmo Genético proposto.

\section{CONCLUSÕES}

Neste trabalho foi apresentada uma implementação da metaheurística Algoritmos Genéticos para resolver o Problema de Rodízio de Tripulações referente a uma empresa que atua no Sistema de Transporte Público Brasileiro. O problema foi dividido em duas etapas: a primeira etapa consiste na utilização dos Algoritmos Genéticos para gerar soluções que definem os padrões de folga, e a segunda etapa consiste na alocação das jornadas propriamente ditas, as quais devem ser realizadas por cada tripulação nos seus respectivos dias de trabalho. A alocação das jornadas é realizada a partir da solução gerada na primeira etapa, que define os dias de folga e a quantidade de tripulações necessárias. Além disso, o problema foi dividido em quatro turnos independentes gerando quatro novos subproblemas: um para cada turno. Para cada subproblema, foram consideradas suas restrições operacionais e trabalhistas. A metaheurística foi modelada para resolver um caso prático da realidade brasileira.

A metaheurística Algoritmos Genéticos foi calibrada, variando-se seus parâmetros com o objetivo de obter a combinação de parâmetros que gerasse os melhores resultados para cada subproblema e etapa considerada. A partir da combinação de parâmetros para cada subproblema e etapa, cinco testes foram executados. Posteriormente, quatro combinações foram selecionadas, visto que apresentaram os melhores resultados em relação ao valor da Função Objetivo. Após a execução de 5 novos testes para cada combinação foi escolhida, para cada subproblema e etapa do PRT, a melhor combinação de parâmetros e as melhores soluções do problema. No total, foram executados 640 testes computacionais e foram selecionadas oito combinações de parâmetros que, de acordo com a análise estatística, devem produzir os melhores resultados para os quatro subproblemas das duas etapas de resolução do PRT.

$\mathrm{O}$ algoritmo foi testado com os dados reais de uma empresa brasileira e mostraram que a solução produzida foi capaz de reduzir em 55\% o total de tripulações necessárias para a execução de todas as jornadas, comparado à escala realizada pela empresa. Além disso, verificou-se também que o modelo proposto obtém a compensação ótima entre as horas extras e ociosas de cada turno. Em relação ao número de tripulações, o Algoritmo Genético ficou a $0,91 \%$ da solução ótima, o que mostra ser um algoritmo bastante eficiente na resolução do problema. Os resultados mostram também que houve uma excelente distribuição da carga de 
trabalho para as tripulações, visto que o Desvio Padrão da carga de trabalho variou entre 1,9 e 137,2 minutos em um horizonte de sete semanas.

\section{AGRADECIMENTOS}

Os autores agradecem à CAPES, ao CNPq, à FAPEMIG e à UFOP pelo apoio recebido no desenvolvimento deste trabalho.

\section{REFERÊNCIAS}

Ahuja, R. K., Magnanti, T. L. e Orlin, J. B. (1993) Network Flows: Theory, Algorithms, and Applications. Prentice Hall, New Jersey.

Bianco, L., BieIli, M., Mingozzi, A., Ricciardelli, S. e Spadoni, M. (1992) A heuristic procedure for the crew rostering problem, European Journal of Operations Research, 58(2), p. 272-283. DOI:10.1016/0377-2217(92)90213-S

Boas, M. G. V. e Silva, G. P. (2014) Modelos de Programação Inteira para o Problema de Rodízio de Tripulações de Ônibus Urbano. Anais do XLVI Simpósio Brasileiro de Pesquisa Operacional, p. 1-12.

Caprara, A., Monaci, M. e Toth, P. (2003) Models and algorithms for a staff scheduling problem, Mathematical Programming, 98(1-3), p. 445-476. DOI:10.1007/s10107-0030413-7

Carraresi, P. e Gallo, G. (1984) A multi-level bottleneck assignment approach to the bus drivers rostering problem, European Journal of Operational Research, 16(2), p. 163-173. DOI: 10.1016/0377-2217(84)90071-7

Ernst, A. T.; Jiang, H.; Krishnamoorthy, M. e Sier, D. (2004) Staff scheduling and rostering: A review of applications, methods and models, European Journal of Operational Research, 153, p. 3-27. DOI:10.1016/S0377-2217(03)00095-X

Goldberg, D. E. (1989) Genetic Algorithms in Search, Optimization and Machine Learning. Addison-Wesley, Berkeley.

Holland, J. H. (1975) Adaptation in Natural and Artificial Systems. University of Michigan Press.

Kruskal, W. H.. e Wallis, W. A. (1952) Use of ranks in the one criterium variance analysis, Journal of American Statistical Association, 47(260), p. 583-621.

DOI:10.1080/01621459.1952.10483441

Leite, P. V. S. (2012) Resolução do Problema de Rodízio de Tripulações Usando um Modelo de Fluxo em Redes. Trabalho de Conclusão de Curso, Departamento de Engenharia de Controle e Automação, Universidade Federal de Ouro Preto.

Mayrink, V. T. M. e Silva, G. P. (2013) Otimização do rodízio de tripulações do sistema de transporte público, Journal of

Transport Literature, 7(3), p. 192-213. DOI:10.1590/S223810312013000300009

Silva, G. P. e Prates, R. F. C. (2014) Otimização da Escala Mensal de Motoristas de Ônibus Urbano Utilizando a Metaheurística Variable Neighborhood Search, TRANSPORTES, 22(1), p. 31-43. DOI:10.14295/transportes.v22i1.698
Talbi, E. G. (2009) Metaheuristics: from design to implementation. John Wiley \& Sons.

Toffolo, T. A., Souza, M. J. F. e Silva, G. P. (2005) Resolução do Problema de Rodízio de Tripulações de Ônibus Urbano via Simulated Annealing e Iterated Local Search, Anais do XIX Congresso de pesquisa e Ensino em Transportes, ANPET, 2, p. $657-668$

Whitley, D. (1994) A Genetic Algorithm Tutorial, Computer Science Department, Colorado State University, Fort Collins, CO 80523. DOI: 10.1007/BF00175354 